\title{
A novel optical flow method for myocardial deformation analysis from tagged MRI
}

\author{
Mohammadreza Negahdar*, Hui Wang, Amir Amini \\ From 15th Annual SCMR Scientific Sessions \\ Orlando, FL, USA. 2-5 February 2012
}

\section{Background}

Cardiac motion analysis can play an important role in diagnosis of cardiac disease. Tagged magnetic resonance imaging (MRI) has the ability to directly and non-invasively alter tissue magnetization and produce tag patterns on the deforming tissue [1-3]. This abstract proposes a novel optical flow method for computing the left ventricular systolic dynamics using harmonic phase (HARP) [4] images extracted from tagged MRI data.

\section{Methods}

Tagged MRI gives rise to spectral peaks in k-space, each peak containing information about motion in a particular direction. Harmonic images are produced by filtering the spectral peaks in the Fourier domain and extracting the spatial phase information from the inverse Fourier transform of the filtered images $[4,5]$.

The horizontal and vertical components of the optical flow field are jointly estimated from harmonic phase images in the two directions. The basic assumption in standard optical flow estimation is the grey value constancy. In the proposed approach, we additionally include gradient constancy and mass conservation which are applied to the image globally, and spatio-temporal smoothness which is applied in a local fashion. For more details, please refer to [6].

\section{Results}

Our method has been applied to both simulated data ground-truth motion fields with SinMod [5] and the proposed method.

\section{Conclusions}

We have presented a novel optical flow method for cardiac motion tracking. Four physical constraints were incorporated into the optical flow energy function to obtain a robust and accurate motion field.

Published: 1 February 2012

\section{References}

1. Zerhouni EA, Parish DM, Rogers WJ, Yang A, Shapiro EP: "Human heart: Tagging with MR imaging-a method for noninvasive assessment of myocardial motion". Radiology 1988, 169(1):59-63.

2. Axel L, Dougherty L: "MR imaging of motion with spatial modulation of magnetization". Radiology 1989, 171(3):841-845.

3. Amini A, Prince J: Measurement of cardiac deformations from MRI: Physical and mathematical models. Dordrecht: Kluwer Academic Publishers; 2001.

4. Osman NF, Kerwin WS, Mcveigh ER, Prince J: "Cardiac motion tracking using CINE harmonic phase (HARP) magnetic resonance imaging". Magnetic Resonance in Medicine 1999, 42(6):1048-1060.

5. Arts T, Prinzen FW, Delhaas T, Milles JR, Rossi AC, Clarysse P: "Mapping displacement and deformation of the heart with local sine wave modeling". IEEE Trans. on Med. Imag 2010, 29(5):1114-1123.

6. Negahdar M, Amini A: "Planar deformable motion estimation incorporating mass conservation and image gradient constancy". Int Sym. On Biomedical Imaging (ISBI) 2011, 536-540.

doi:10.1186/1532-429X-14-S1-W45

Cite this article as: Negahdar et al:: A novel optical flow method for myocardial deformation analysis from tagged MRI. Journal of Cardiovascular Magnetic Resonance 2012 14(Suppl 1):W45. and two frames of a canine study together with the computed motion fields are shown in Figure 1. Table 1 reports the angular error (AE) and relative root mean squared (RRMS) error between the calculated and the 

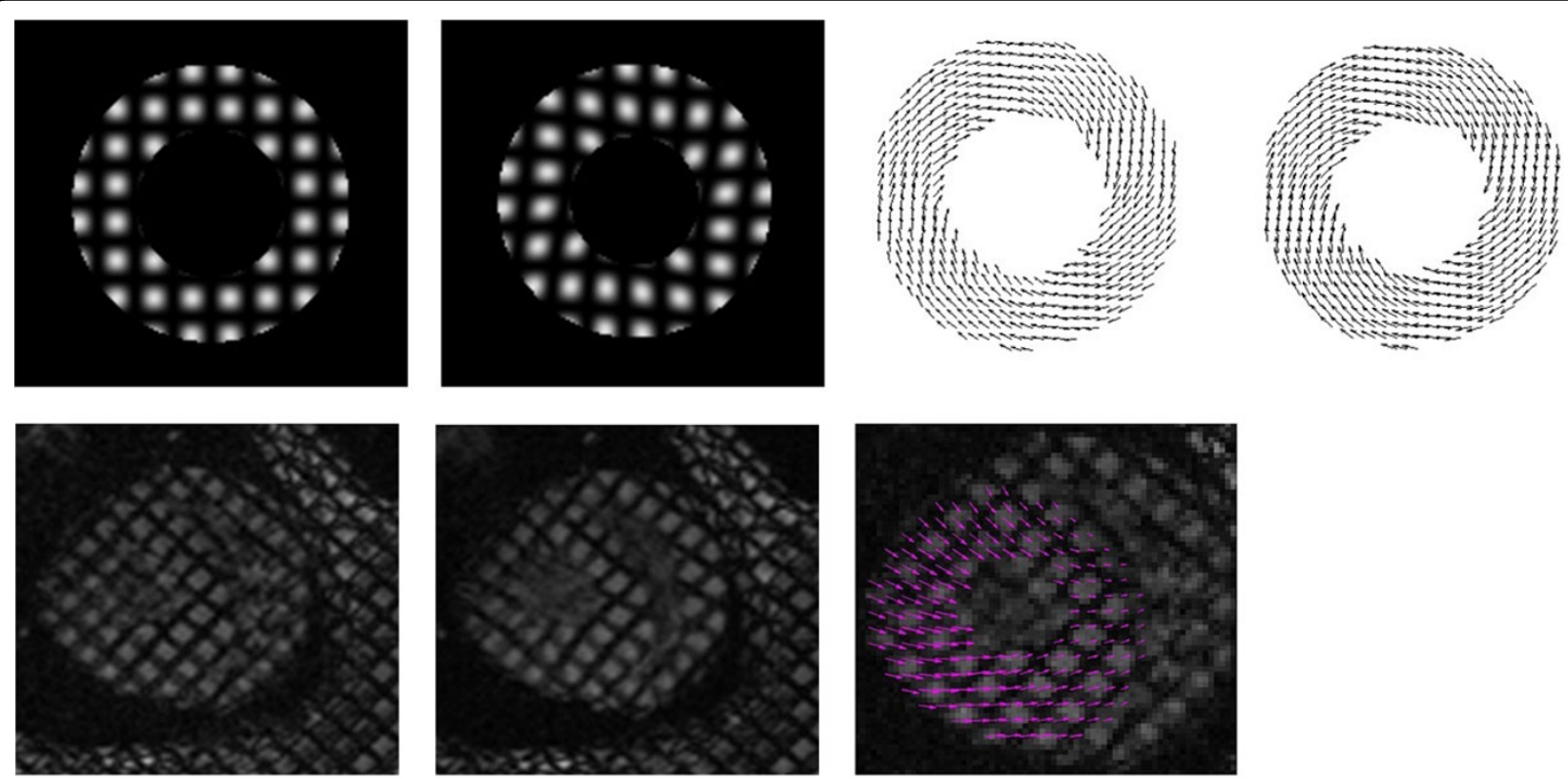

(a)

(b)

(c)

(d)

Figure 1 Tagged MR images and computed motion field between two frames for simulated data (row 1) and canine data (row 2). (a) reference image, (b) deformed image, (c) motion field between reference image and deformed image as computed with the proposed method, (d) ground truth motion field for simulated data.

Table 1 AE and RRMS error between the ground truth motion field (obtained from the cardiac motion simulator) and motion fields calculated with SinMod and the proposed method.

\begin{tabular}{ccccc}
\hline Frame Numbers & \multicolumn{2}{c}{ SinMod } & \multicolumn{2}{c}{ Proposed Method } \\
\hline & AE & RRMS (\%) & AE & RRMS (\%) \\
\hline Frame 1-2 & $9.63^{\circ}$ & 15.40 & $7.62^{\circ}$ & 14.45 \\
Frame 1-3 & $8.55^{\circ}$ & 15.91 & $5.99^{\circ}$ & 12.92 \\
Frame 1-4 & $7.93^{\circ}$ & 15.35 & $6.56^{\circ}$ & 13.66 \\
Frame 1-5 & $8.66^{\circ}$ & 15.43 & $6.90^{\circ}$ & 13.75 \\
\hline
\end{tabular}

